\title{
Integration of Model-Based Signal Control and Queue Spillover Control for Urban Oversaturated Signalized Intersection
}

\author{
Haitao Xu (i), Jing Chen (D), and Jie Xu \\ School of Computer Science and Technology, Hangzhou Dianzi University, Hangzhou, China \\ Correspondence should be addressed to Haitao Xu; xuhaitao@hdu.edu.cn
}

Received 6 March 2019; Revised 14 May 2019; Accepted 2 June 2019; Published 10 July 2019

Academic Editor: Ana Maria A. C. Rocha

Copyright (C) 2019 Haitao Xu et al. This is an open access article distributed under the Creative Commons Attribution License, which permits unrestricted use, distribution, and reproduction in any medium, provided the original work is properly cited.

\begin{abstract}
An improved model-based predictive control approach integrating model-based signal control and queue spillover control is proposed in this paper, which includes three modules: model-based signal control, queue spillover identification, and spillover control to deal with the problem of traffic congestion for urban oversaturated signalized intersection. The main steps are as follows. First of all, according to the real-time traffic flow data, the green time splits for all intersections will be solved online by the modelbased signal control controller whose optimization model is based on model-predictive control (MPC) strategy. Second, the queue spillover identification module will be used to detect the potential queue spillover. If potential queue spillover is detected, the spillover control module will be activated to prevent vehicles from the upstream link of the link with possible spillover from entering the downstream link to avoid traffic congestion. The experiment is performed on a simulated road network. The results verify that the proposed scheme can significantly decrease the delay which reflects the overall performance of the studied intersection.
\end{abstract}

\section{Introduction}

With the rapid development of society and economy, the number of vehicles has grown larger and larger. Urban Signalized intersections are under oversaturated traffic conditions during peak hours more and more frequently. Under this circumstance, the urban traffic congestion becomes more and more serious, resulting in excessive delays and increased travel times. The traffic congestion resulting from oversaturated traffic conditions will bring about significant economic losses and social costs. Therefore, it is necessary to develop an effective control method for the control of urban oversaturated signalized intersection to avoid traffic congestion.

Different solutions including traffic signal control $[1,2]$, route guidance $[3,4]$, continuous network design (CNDP) [5], and different combinations of these strategies $[6,7]$ have been developed to deal with the problem of traffic congestion. Among the above methods, traffic signal control is of great importance to control and manage traffic effectively [8]. Many scholars have done a lot of research work on traffic signal control methods for a long time. As we all know, SCOOT and SCATS are two famous traffic-responsive urban control systems which have been extensively applied in many cities. Liu [9] proposed a method to optimize green phase time, offset, cycle length, and left-turn phase sequence simultaneously. Samra et al. [10] proposed a dynamic programming method to obtain the optimal green time splits. Castro et al. [11] proposed an adaptive biologically-inspired neural network method to control urban traffic. However, they function ineffectively under oversaturated condition [12].

To solve the problem of traffic signal control for oversaturated intersections, extensive efforts have been made. To the best of our knowledge, traffic demand which represents the traffic arrivals on intersection approaches is too high to be managed effectively when the intersection is oversaturated, which may induce queue spillover [13]. Therefore, the first and the most important thing is to avoid queue spillover when the intersections are under oversaturated conditions. Traffic signal control methods under such conditions mainly fall into two categories: offline control methods and online control methods. For offline control method which is termed as pretimed control method, the semigraphical method is proposed to optimize the control of two adjacent oversaturated intersections [14]. Michalopoulos et al. [15] employed a "bang-bang" control model with restrained queue length to 
deal with the problem of optimal timing during the oversaturated period. However, these strategies obtain optimal timing according to historical data rather than real-time data, which is difficult to adapt to the real-time traffic conditions [16]. Therefore, extensive research on online control strategies has been proposed [17].

For online control method which can adjust the timing scheme according to current collected traffic flow, a number of methods have been presented. Lin et al. [18] proposed a quasidynamic robust control method which can balance the proportion of queue length on different roads under oversaturated traffic condition. Ma et al. [19] proposed a bottleneck control method to prevent spillovers under oversaturated traffic conditions, which can adjust the capacity of bottleneck links according to the data of occupancy. Qi et al. [20] developed a virtual signal method to avoid the spillback of channelized section. A simulation-based approach which takes the queue-length information as objective function was proposed by Osorio et al. [21]. Xiang et al. [16] proposed a static state feedback control strategy to relieve the waiting vehicles in lanes. Sun et al. [22] presented a queue-based quasi-optimal feedback control strategy to limit queue length and extended it afterwards [17]. More recently, Ren et al. [23] proposed a method to identify potential spillover conditions and to prevent it periodically. Ren et al. [24] proposed a method based on the connected vehicle environment to identify queue spillover trend and optimized the signal timing. Wu et al. [25] proposed a two-point-three state traffic state perception model to analyze the traffic state and to determine the corresponding green time extension scheme.

Although the aforementioned methods can limit the queue length to mitigate oversaturation to some extent, these methods cannot predict future traffic condition. A modelbased predictive control method which deals with the optimal problem over a finite horizon according to the current traffic flow can address this problem perfectly. Aboudolas et al. [26] proposed a model predictive control method which embeds a store-and-forward model into a rolling-horizon control scheme to calculate the optimal timing plan. To improve the computational complexity further, an improved model predictive control method based on a simplified model [27] was proposed by enlarging the simulation time interval to one cycle time. The simulation results demonstrated that the proposed method can improve significantly the traffic signal control performance. For these methods, it is important to predict the traffic demand over the finite horizon accurately. However, it is difficult to calculate the traffic demand accurately based on the basic model predictive control approach when the queue over detectors is often encountered under oversaturated traffic conditions. Therefore, we propose a novel method to optimize green time splits under such conditions.

The details of the contributions in this paper include two aspects which can be listed as follows. (1) We propose an improved model-based predictive control approach integrating model-based signal control and queue spillover control to obtain an optimal green time allocation scheme and to avoid spillover simultaneously under oversaturated traffic conditions. (2) Considering that the objective of this model is complicated when the model is optimized in several cycles, we propose an improved hybrid artificial bee algorithm to address the optimal control problem of the proposed improved model-based predictive control approach as soon as possible.

The rest of the paper is organized as follows. Section 2 describes the control framework of the proposed method. Section 3 includes the prediction model, optimization model, and optimization algorithm for model-based signal control controllers. The control method to detect the possible spillover is given in Section 4. The queue dissipation identification and spillover control method is introduced in Section 5. Simulation evaluation results of a case study and the comparative analysis of the proposed control scheme with other methods are provided in Section 6. Recommendations for future research and conclusions are provided in Section 7.

\section{System Framework}

In this paper, we proposed an improved model-based predictive control method which can simultaneously optimize green time splits according to the predicted traffic demand over a finite horizon and manage queue based on different traffic flow conditions. The framework of the proposed method is shown in Figure 1.

Figure 1 shows that the control architecture mainly consists of three parts: model-based signal control, queue spillover identification, and spillover control. The modelbased signal control method is designed to deal with the optimal control problem based on the control strategy of the basic model predictive control, which contains a prediction model to predict the future traffic flow over a given horizon. The optimization model of model predictive control with the control objective of minimizing the total travel time can be constructed according to the prediction results. A new IGAABC algorithm is proposed to obtain the optimal solutions of the optimization model. The part of queue spillover identification and spillover control can adaptively regulate the green time of the relevant signal phases encountering possible queue spillover.

In order to control the studied intersection, the corresponding traffic data should be collected by some detectors. The information of queue length and the number of vehicles in link $l(u, d)$ (for all $l \in L$, where $L$ is the set of roads) collected from the road at the start of each cycle is used as the input of model-based signal control controller. The information of real-time vehicle speed and vehicle counts can be used to identify queue spillover and dissipation. Assume that the cycle time for all intersections is equal to the sampling time interval. The process of the proposed integrated control scheme will be formulated in detail in the following.

At the beginning of each signal cycle, the model-based signal control method will firstly be applied to obtain an optimal control sequence (green time splits for each phase at all intersections) with the objective of minimizing the total travel time according to the current traffic flow information including the number of vehicles and queue length for each link. Then the queue spillover identification method will be activated to identify the possible queue spillover according 


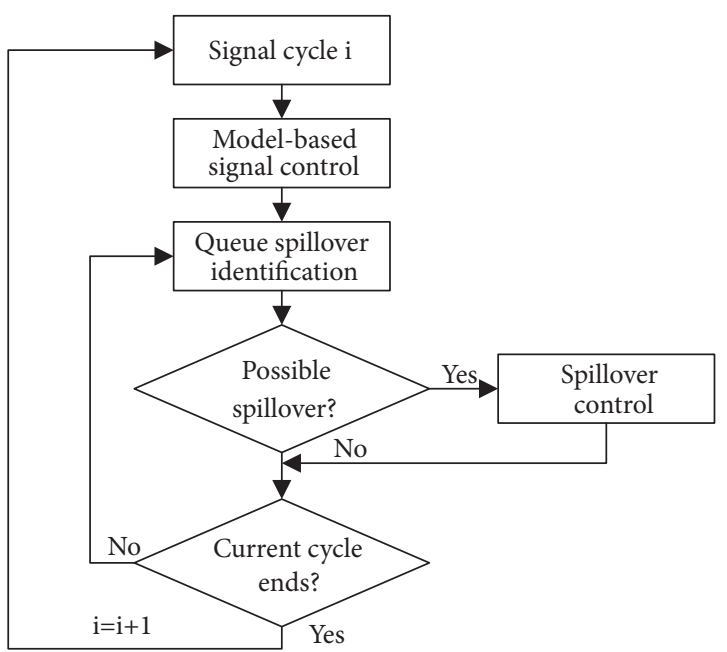

Figure 1: Framework of the proposed method. The proposed method mainly includes three modules: model-based signal control, queue spillover identification, and spillover control.

to the real-time traffic flow information (the speed and the vehicle count) which is obtained by the detector periodically and the current traffic signal status. The spillover control method will be used to prevent the potential queue spillover if potential queue spillover is identified. When there is no potential queue spillover on the downstream link, the system will further determine whether the current control cycle should be terminated. If yes, the next cycle will be executed; otherwise, the queue spillover identification method will continue to detect the possible spillover.

\section{Model-Based Signal Control}

Suppose that the signal phase and phase sequence is fixed at each intersection. At every control moment, the model-based signal control method will provide the optimal green time splits at each intersection on the basis of the information of current traffic flow. Accordingly, we should choose an appropriate prediction model to predict traffic flow dynamics and an appropriate optimization model to reflect the optimization objective of traffic control. Finally, a novel optimization algorithm is developed for obtaining solutions of the optimization model. We will first introduce the prediction model and optimization model, respectively [28], and then explain the optimization algorithm.

\subsection{Prediction Model}

3.1.1. Traffic Data Collection. The model-based signal control method will be executed to obtain the optimal green time splits periodically according to the current number of vehicles and queue length for each link. Therefore, it is necessary to configure the corresponding detectors to detect traffic flow data to provide the input for the model-based signal control controller. The detectors can be deployed after the stop bars for all intersections to detect the queue length and

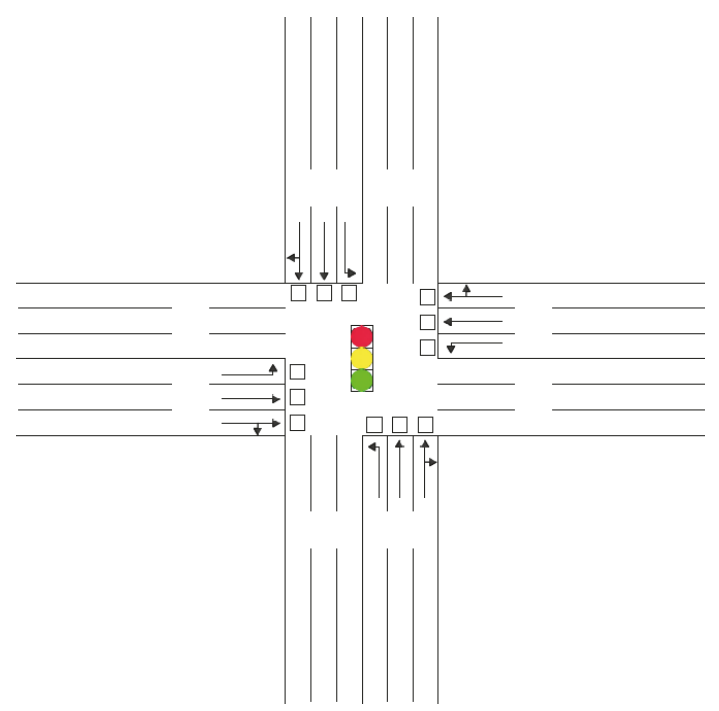

Figure 2: Detector configuration at intersection 1. Four queue detectors are deployed over the stop bar at the intersection where arrows represent the corresponding turning movement.

the number of vehicles in all links at the start of every traffic signal cycle periodically. As shown in Figure 2, the black rectangle at intersection 1 denotes the corresponding lane which should be deployed with a queue counter detector. The configurations of other intersections are similar.

3.1.2. Prediction Model Description. The S model as a simplified macroscopic traffic model initially proposed by Lin [29], which is then applied to model a complicated traffic network by Zhou et al. [28], can be used to model the evolution of urban traffic in both unsaturated and oversaturated traffic situations. It is an appropriate model which can take account of both computation time and accuracy. What is more, we expand the prediction time interval of this model to one cycle which is of great importance to reduce the computation time and guarantee a better performance simultaneously.

As shown in Figure 3, assume that a road network including two intersections is composed of roads and intersections. We define the set of all intersections and links by $I$ and $L$, respectively. Link $(u, d)$ belonging to the set of $L$ denotes the link which is between the intersection $u$ and the corresponding intersection $d$. The collection of upstream intersections of link $(u, d)$ can be denoted by $I_{u, d}$, while the collection of downstream intersections of link $(u, d)$ can be denoted by $O_{u, d}$.

Suppose that $c_{c y c l e}$ represents the control cycle of signal controller. The sampling time $T$ of queue counter detectors is equal to $c_{c y c l e}$. According to the value of $n_{u, d}(k)$ collected by link evaluation on link at time step $k$, the number of vehicles at time step $k+1$ can be predicted as follows:

$$
n_{u, d}(k+1)=n_{u, d}(k)+\left(\alpha_{u, d}^{\text {enter }}(k)-\alpha_{u, d}^{\text {leave }}(k)\right) \cdot c_{c y c l e}
$$




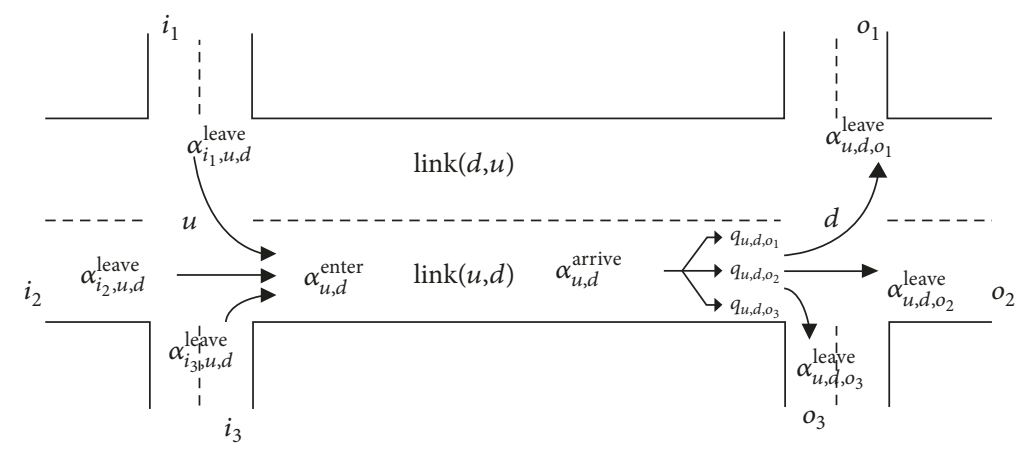

FIGURE 3: A road network including two intersections. The evolution process of traffic flow at two adjacent intersection is demonstrated.

where $\alpha_{u, d}^{\text {enter }}(k)$ which represents the flow rate at time step $k$ when the vehicles enter link $(u, d)$ from different upstream intersection can be calculated as below:

$$
\alpha_{u, d}^{\text {enter }}(k)=\sum_{i \in I_{u, d}} \alpha_{i, u, d}^{\text {leave }}(k)
$$

in which $\alpha_{u, d}^{\text {leave }}(k)$ is the flow rate at time step $k$ when the vehicles leave link $(u, d)$ to different downstream links, that is,

$$
\alpha_{u, d}^{\text {leave }}(k)=\sum_{o \in \mathrm{O}_{u, d}} \alpha_{u, d, o}^{\text {leave }}(k)
$$

$\alpha_{u, d, o}^{\text {leave }}(k)$ which represents the leaving flow rate of vehicles waiting on link $(u, d)$ to the intersection $o$ at time step $k$ is equal to the minimum value of three terms which can be calculated as below:

$$
\begin{gathered}
\alpha_{u, d, o}^{\text {leave }}(k)=\min \left(\beta_{u, d, o} \cdot \mu_{u, d} \cdot \frac{g_{u, d, o}(k)}{c_{c y c l e}}, \frac{q_{u, d, o}(k)}{c_{c y c l e}}\right. \\
\left.+\alpha_{u, d, o}^{\text {arrive }}(k), \frac{\beta_{u, d, o}\left(C_{d, o}-n_{d, o}(k)\right)}{c_{c y c l e}}\right)
\end{gathered}
$$

where the first term is the free flow rate where vehicles can pass an intersection with a saturation flow rate $\mu_{u, d}$. The second is the rate releasing the vehicles in the queue. The last one is the releasing rate when there is not enough room to accommodate vehicles in link $(d, o) . \beta_{u, d, o}$ represents the proportion of the vehicles entering the intersection o. The green time splits which we intend to optimize can be defined as $g_{u, d, o}(k)$. We denote the number of vehicles that link $(d, o)$ can accommodate by $C_{d, o}$.

The queue length of different lanes at time step $k+1$ can be predicted as below, where $\alpha_{u, d, o}^{\text {arrive }}(k)$ is the arriving rate from the entrance of link $(u, d)$ to the lane on which vehicles can turn to intersection $o$.

$$
\begin{aligned}
q_{u, d, o}(k+1)= & q_{u, d, o}(k)+\left(\alpha_{u, d, o}^{\text {arrive }}(k)-\alpha_{u, d, o}^{\text {leave }}(k)\right) \\
& \cdot c_{c y c l e}
\end{aligned}
$$

The total queue length can be calculated as below:

$$
q_{u, d}(k)=\sum_{o \in O_{u, d}} q_{u, d, o}(k)
$$

According to the corresponding $\beta_{u, d, o}$, we can calculate the arriving rate when vehicles join different queues of link $(u, d)$ as below:

$$
\alpha_{u, d, o}^{\text {arrive }}(k)=\beta_{u, d, o} \cdot \alpha_{u, d}^{\text {arrive }}(k)
$$

where $\alpha_{u, d}^{\text {arrive }}(k)$ is determined by the formula (8)

$$
\begin{aligned}
\alpha_{u, d}^{\text {arrive }}(k)= & \left(1-\xi_{u, d}(k)\right) \cdot \alpha_{u, d}^{\text {enter }}\left(k-\eta_{u, d}(k)\right) \\
& +\xi_{u, d}(k) \cdot \alpha_{u, d}^{\text {enter }}\left(k-\eta_{u, d}(k)-1\right)
\end{aligned}
$$

where $\eta_{u, d}(k)$ is an integral part of $\sigma_{u, d}(k)$ divided by $c_{c y c l e}$, while $\xi_{u, d}(k)$ is the remainder of it, as shown in the following two formulas:

$$
\begin{aligned}
& \eta_{u, d}(k)=\left\lfloor\frac{\sigma_{u, d}(k)}{c_{c y c l e}}\right\rfloor \\
& \xi_{u, d}(k)=\operatorname{rem}\left\{\sigma_{u, d}(k), c_{c y c l e}\right\}
\end{aligned}
$$

where $\sigma_{u, d}(k)$ is the time the traffic flow takes to join the queue from the entrance of link $(u, d)$ which can be calculated according to formula (11). $l_{v e h}$ represents the average vehicle length. $N_{u, d}^{\text {lane }}$ represents the number of lanes of link $(u, d)$. Vehicles would move with a normal speed $v_{u, d}^{\text {free }}$ under unobstructed traffic conditions.

$$
\tau_{u, d}(k)=\frac{C_{u, d}-q_{u, d}(k) \cdot l_{v e h}}{N_{u, d}^{l a n e} \cdot v_{u, d}^{f r e e} \cdot c_{c y c l e}}
$$

3.2. Optimization Model. To obtain optimal green time splits according to varied traffic flow, it is essential to select an appropriate optimization model which can balance the computation time and accuracy. The model described in [28] is used as the optimization model which can be embedded in a rolling-horizon framework. The appeal of this model is that it can provide signal timing scheme according to the 
predicted traffic flow data, which is good for avoiding myopic control. The optimization model is described as follows:

$$
\begin{aligned}
& \min _{g(k)} \sum_{(u, d) \in L} \sum_{p=1}^{N_{p}} n_{u, d}(k+p) \cdot c_{c y c l e} \\
& \text { s.t. } \quad n_{u, d}(k+p+1)=f\left(n_{u, d}(k+p), g_{d}(k+p)\right) \\
& \qquad \text { for } p=0, \ldots, N_{p}-1
\end{aligned}
$$

$g_{\min } \leq g(k) \leq g_{\max }$

We regard formula (12) which represents the total time spent as the optimization objective to obtain preferable green time splits $g(k)$, where $N_{p}$ is the prediction horizon which reflects the number of time steps of the prediction model.

According to the queue length and the number of vehicles in all links at time step $k$, formula (13) is regarded as the prediction model that can predict the future traffic flow for each road in the simulation network, which is presented in Section 3.1 in detail.

Formula (14) ensures that the sum of green timing splits at each intersection is equal to $c_{c y c l e}$, in which $H_{d}$ represents all the traffic signal phases of the corresponding intersection. $g_{d, h}(k)$ represents the green time of the corresponding phase at intersection $d$. All intersections belonging to the set of $I$ should subject to this constraint.

Formula (15) limits green time range within $\left[g_{\min }, g_{\max }\right]$, where upper and lower bound denote minimum and maximum time, respectively, for each phase at different intersections.

To obtain the solutions of the above optimization model online, we will propose an appropriate optimization algorithm. It will be illustrated in the following.

\subsection{Optimization Algorithm}

3.3.1. Artificial Bee Colony (ABC) Algorithm. The ABC algorithm is proposed to solve optimization problems in many different fields, including unconstrained optimization problems [30], constrained problem optimization [31], and cluster analysis [32]. Recently, this algorithm has been successfully applied to the field of transportation [33]. The ABC algorithm with fewer parameters is relatively easy to implement so that a lot of researchers have studied this algorithm to deal with various problems.

Suppose that both the size of employed bees and the onlooker bees are $S N$. This algorithm first generates $S N$ solutions with $\mathrm{D}$-dimensional variables between upper and lower bounds randomly. Secondly, every employed bee will update the solutions. If the fitness value of the new solution is better, then the solutions will be replaced. And then, onlooker bees will select the employed bee to follow according to the selection probability. Finally, if the solutions cannot be

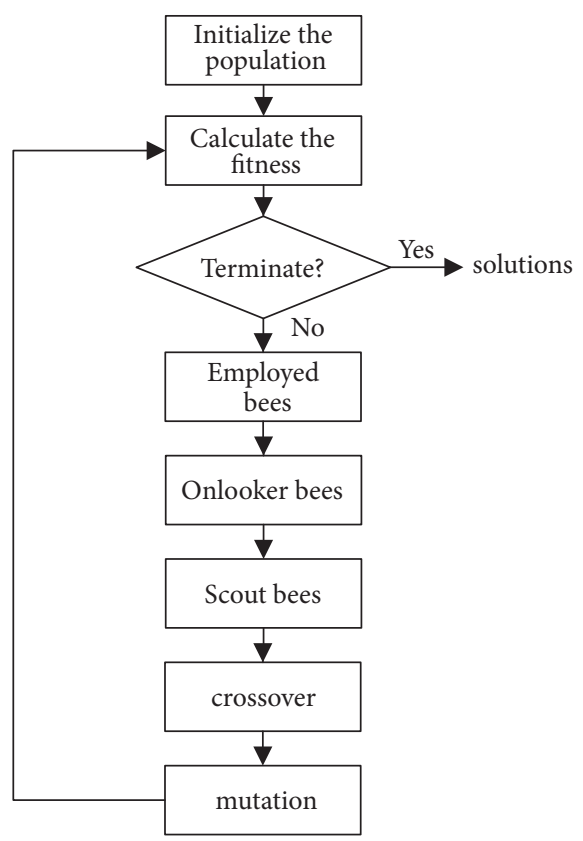

FIGURE 4: The flowchart of the IGAABC. The operators of crossover and mutation are introduced into the basic $\mathrm{ABC}$ algorithm to avoid getting trapped in local optima.

further improved in less than trials_limit times, it will be abandoned. Employed bee will be transferred to a scout bee and scout bee phase will be executed.

3.3.2. An Improved Hybrid Artificial Bee Algorithm (IGAABC). Although the $\mathrm{ABC}$ algorithm is simple enough to obtain optimal solutions of complicated problems, it tends to get trapped in local optima. Therefore, a number of scholars studied ABC algorithm and developed various methods to deal with this deficiency which arises from the reduction of diversity of bee population. Zhu et al. [34] propose an improved $\mathrm{ABC}$ algorithm to promote the performance of exploitation. Gao et al. [35] improve ABC algorithm by taking advantage of the chaotic maps where the relatively scattered chaotic sequence can traverse the corresponding interval to the population initialization to avoid premature convergence. Ma et al. [36] develop a modified ABC algorithm which adds the disturbance to avoid getting trapped in local optima.

In order to obtain better solutions, we propose an IGAABC algorithm to overcome the deficiency of premature convergence and slow convergence rate of the basic $\mathrm{ABC}$ algorithm. The preceding few steps of this algorithm are analogous to $\mathrm{ABC}$ algorithm except that the fitness function of $\mathrm{ABC}$ algorithm is modified. After the operations of the scout bee stage are finished, we will add the steps of crossover, mutation operation to improve the basic algorithm. The flowchart of the improved hybrid artificial bee colony optimization for the optimization of signal timing is shown in Figure 4, and the details of IGAABC will be introduced next.

It is well known that Genetic Algorithm (GA) [37] inspired by the acts of biological evolution is an excellent 


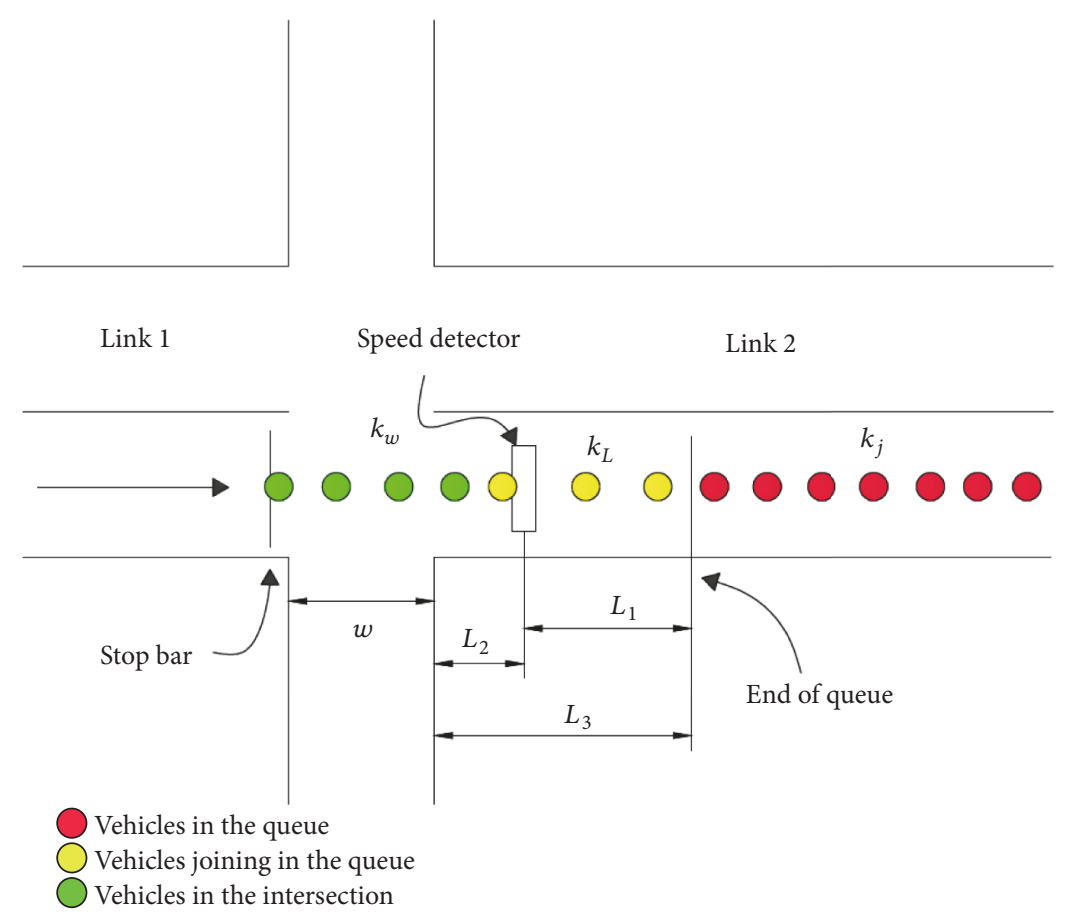

FIGURE 5: Queue spillover identification based on speed detector. Speed detector is deployed to detect the speed when vehicles pass over it.

optimization tool. The processes of GA algorithm are as follows. Firstly, the algorithm will generate an initial population, which consists of a group of initial solution in feasible domain of the specific problem. Second, the fitness of each solution in the population will be calculated. Finally, the operators of selection, crossover, and mutation will be performed. Individuals can be selected according to their fitness in the process of selection operators which is consistent with the law of survival of the fittest. Two individuals will refer to the defined crossover probability $p_{c}$ to determine whether or not to execute crossover. Similarly, the individual will execute mutation operators according to the mutation probability $p_{m}$. Repeat the process of selection, crossover, and mutation until the termination conditions are met. Considering that the operators of crossover and mutation can increase the diversity of solutions, we introduce these two operators into $\mathrm{ABC}$ algorithm to avoid getting trapped in local optima. Due to the green time splits we intend to optimize is decimal, we should choose a method to crossover in decimal. There are two ways to crossover in decimal. One is to convert to binary crossover and then convert the value to decimal. The other is to directly cross in decimal. The method of direct crossover is further divided into single point crossover, fixed, and random multipoint crossover [38]. The method of decimal random multipoint crossover is selected as the crossover methods in our algorithm. The details of this method will be introduced as follows.

First, two parents will be selected from the original population. Secondly, the algorithm will generate the number $b$ within $[0,1]$ randomly. Define the crossover probability as $p_{c}$. If $b<p_{c}$, these two individuals will be crossed according to the method of random multi-point crossover. Otherwise, it is determined whether to cross the other two individuals. The process of random multi-point crossover is as follows.

(1) Generate a vector y containing D "0 or 1" randomly, where $\mathrm{D}$ is equal to the dimension of solutions. Each element of $y$ corresponds to the individual's gene (decimal number).

(2) The position whose value is equal to one in y of these two individuals will be crossed as below:

$$
\begin{aligned}
& S_{i}^{\prime}=\operatorname{round}\left[r S_{i}+(1-r) S_{j}\right] \\
& S_{j}^{\prime}=\operatorname{round}\left[r S_{j}+(1-r) S_{i}\right]
\end{aligned}
$$

where $r$ is a number generated randomly within the interval $[0,1] . S_{i}, S_{j}$ are two individuals performing the crossover. $S_{i}^{\prime}, S_{j}^{\prime}$ are new individuals produced by the crossover.

(3) After these two individuals finish crossing, we transfer to the crossover of the other two individuals.

The mutation operator of our algorithm is similar to GA's. The flowchart of the IGAABC algorithm is shown in Figure 4.

The selection of the fitness function directly affects the convergence speed and determines whether the optimal solution can be found because the performance of the solution is evaluated on the basis of the fitness function. Therefore, it is important to design an appropriate fitness function to determine whether a solution is good or bad so that an optimal solution can be obtained within a limited number of iterations. So we modified the fitness function of the artificial bee algorithm as follows:

$$
\text { fitness }_{i}= \begin{cases}\frac{1}{1+f_{i}^{2}} & f_{i} \geq 0 \\ f_{i}^{2} & f_{i}<0\end{cases}
$$


where $f_{i}$ represents the optimal target which reflects the total travel time in this paper. The fitness function fitness $s_{i}$ can be calculated based on the above formula (17). If $f_{i} \geq 0$, the result is equal to the inverse of $1+f_{i}^{2}$. This modification can increase the rate of change of solution which will be beneficial to speed up the convergence rate.

\section{Queue Spillover Identification}

Extensive research on queue length based method, occupancy based method, and velocity based method has been carried out to identify queue spillover accurately and timely. In terms of the method based on queue length, it is important to estimate queue length accurately. The queue length estimation mechanisms are analyzed to estimate the queue length based on different layout strategies [39]. To detect queue spillover according to the velocity of vehicles, Ren et al. [23] developed an adaptive signal control method which can prevent traffic jams effectively at a specific intersection under oversaturated traffic conditions. In this paper, we adopt the method based on the velocity of vehicles to identify queue spillover.

To identify the spillover for each direction of an intersection, it is necessary to add some detectors to the corresponding link. As shown in Figure 5, in order to identify the queue spillover of Link 2, Link 2 should be deployed with a data collection detector named speed detector to detect realtime vehicle speed and a stop bar detector to detect the vehicle counts. Vehicles will accumulate in Link 2 if the traffic signal for the relevant phase in Link 2 is red. We denote the width of Intersectionl by $w . L_{1}$ is the distance between the speed detector and the end of queue. $L_{2}$ is the distance between the entrance of the link and the deployed speed detector. $L_{3}$ is the distance between the entrance of the link and the end of queue.

According to geometric relationships in Figure 5, we can conclude

$$
L_{3}=L_{1}+L_{2}
$$

The possible queue spillover will be detected by comparing the information collected by the speed detector with the critical speed at which the spillover will happen. We will introduce the process to obtain the critical value in the following.

If the vehicle count which Link 2 can accommodate is too little to receive the vehicles from the upstream link, the spillover will occur. Assume that $k_{j}$ is the density when Link 2 is completely occupied with cars. $k_{w}$ and $k_{L}$ are defined as the vehicle density at different positions. We have

$$
L_{3} k_{j} \leq\left(w+L_{2}\right) \cdot k_{w}+L_{1} k_{L}
$$

According to relationships $L_{3}=L_{1}+L_{2}$ and the formula (19), we can obtain the range of $L_{1}$.

$$
0 \leq L_{1} \leq \frac{k_{w}\left(w+L_{2}\right)-k_{j} L_{2}}{k_{j}-k_{L}}
$$

Assume that the vehicles entering from the entrance of link 2 begin to decelerate when passing over the speed detector if the vehicles queue in Link 2. These vehicles must stop within distance $L_{1}-L_{1}\left(k_{L} / k_{j}\right)$ to avoid rearend collision. Therefore, the breaking distance which can be denoted as follows must be less than $L_{1}-L_{1}\left(k_{L} / k_{j}\right)$ :

$$
f(v, a(t)) \leq L_{1}-L_{1} \frac{k_{L}}{k_{j}}
$$

where $v$ and $a(t)$ are the speed and deceleration rate of the vehicle through speed detector respectively. However, $a(t)$ is difficult to calculate because it is not constant when the vehicles join the queue. It is necessary to estimate it accurately. The average deceleration rate between the stop bar detector and the speed detector is used as the approximation of $a(t)$. Define $t_{1}$ and $t_{2}$ as the times through these two detectors which can collect the information of travel time, $v_{1}$ and $v_{2}$ as the corresponding speeds. The average deceleration rate is estimated as follows:

$$
a(t)=\frac{v_{2}-v_{1}}{t_{2}-t_{1}}
$$

Supposing the deceleration rate is constant which is denoted by $a$, according to kinematics theory, we have $f(v, a)=v^{2} / 2|a|$. Combining the above formulae (20) and (21), we have

$$
f(v, a)=\frac{v^{2}}{2|a|} \leq \frac{k_{w}\left(w+L_{2}\right)-k_{j} L_{2}}{k_{j}}
$$

According to the above formula (20), we can conclude that $k_{\omega}\left(\omega+L_{2}\right)-k_{j} L_{2} \geq 0$, since $k_{j}$ is larger than $k_{L}$, and $L_{1}$ is always nonnegative. Thus (23) can be modified as below:

$$
v \leq \sqrt{2|a| \frac{k_{w}\left(w+L_{2}\right)-k_{j} L_{2}}{k_{j}}}
$$

Therefore, we define critical speed as below:

$$
v_{t h}=\sqrt{2|a| \frac{k_{w}\left(w+L_{2}\right)-k_{j} L_{2}}{k_{j}}}
$$

In view of that it is difficult to obtain the parameter of $k_{w}$, we further simplify the equation (25). We denote the vehicle counts at stop bar detector by $N_{b}$ which can be detected by the corresponding detectors. Similarly, $N_{s}$ is the vehicle counts at the speed detector. Therefore, when $\left(N_{b}-N_{s}\right) / k_{j}-L_{2}$ is nonnegative, the threshold speed can be calculated as follows:

$$
\begin{aligned}
v_{t h} & =\sqrt{2|a| \frac{N_{b}-N_{s}-k_{j} L_{2}}{k_{j}}} \\
& =\sqrt{2|a|\left(\frac{N_{b}-N_{s}}{k_{j}}-L_{2}\right)}
\end{aligned}
$$


Otherwise, the queue spillover will not occur unless the vehicle speed passing over the speed detector is nonpositive, that is,

$$
\begin{aligned}
& v_{t h} \\
& = \begin{cases}0 & \text { if } \frac{N_{b}-N_{s}}{k_{j}}-L_{2}<0 \\
\sqrt{2|a|\left(\frac{N_{b}-N_{s}}{k_{j}}-L_{2}\right)} & \text { if } \frac{N_{b}-N_{s}}{k_{j}}-L_{2} \geq 0\end{cases}
\end{aligned}
$$

Considering the disturbance in vehicle speed in real life, the method to identify the possible queue spillover can be expanded as below.

(1) The speed which is less than the critical speed $v_{t h}$ can be detected by two consecutive samplings.

(2) The speed of vehicles is decreasing.

\section{Spillover Control}

When potential queue spillover is detected, the spillover control method will prevent the upstream vehicle from entering the downstream link to avoid the queue spillover until the queue dissipation is detected. Therefore, we will introduce the method to identify the queue dissipation and control the spillover in the following.

5.1. Queue Dissipation Identification. When the queue dissipation [23] is detected by the corresponding detector on Link 2 , we should ensure that there is enough space on Link 2 to receive the vehicles entering from the upstream link [31]. Thus it is necessary to determine the position of the speed detector to guarantee the space on Link 2 is enough when the detected speed is larger than 0 . The vehicles on Link 2 begin to release when the vehicle queue is served by green light, as shown in Figure 6.

The released capacity $\Delta L$ on Link 2 can be calculated according to geometric relationships.

$$
\Delta L=\widetilde{l}-\widetilde{l} \frac{k_{f}}{k_{j}}
$$

where $k_{f}$ represents the vehicle density at the corresponding position as shown in Figure 6. $\tilde{l}$ is the distance between the stop bar and the frontier of queue. $\widetilde{L}$ is the distance between the stop bar and the Speed detector. We can conclude the relationship that $\tilde{l} \leq \tilde{L}$.

When the vehicles waiting on Link 1 are served by the green time, the number of vehicles that will enter the intersection is more than $s * g_{\text {min }}$ which represents the minimum number of released vehicles. When the queue dissipation is detected by the speed detector, the capacity on Link 2 should be large enough to accommodate the minimum number of released vehicles at least. That is, $\Delta L \cdot k_{j} \geq s * g_{\min }$ which can be simplified as below:

$$
\tilde{l} \geq \frac{s * g_{\min }}{k_{j}-k_{f}}
$$

We can calculate $L_{2}$ by the following:

$$
L_{2}=L-\tilde{L} \leq L-\tilde{l} \leq L-\frac{s * g_{\min }}{k_{j}-k_{f}}
$$

Therefore, the speed detector can be deployed near the downstream entrance as possible to detect both queue spillover and dissipation which is consistent with Section 4. In order to avoid the fluctuation of vehicle speed, the following two conditions can be used to identify the occurrence of queue dissipation.

(1) The speed which is larger than zero can be detected by two consecutive samplings.

(2) The speed of vehicles is increasing.

5.2. Spillover Control. The spillover control method can be activated when the possible queue spillover occurs [23]. This control method consists of two kinds of control scheme, namely, ECG (Early Cut-Off Green) and RUG (Return Unused Green). When the possible queue spillover is detected, the ECG control scheme will first be executed to prevent possible spillover. When the queue dissipation is detected, the (Return Unused Green) will be activated to give back the remaining green time to the phase which has executed the ECG (Early Cut off Green) control scheme if the current control cycle is not ended. The flowchart of queue spillover control on link $L_{1,2}$ is shown in Figure 7 .

Assume Intersection 1 is the studied intersection as shown in Figure 8. Both of these two intersections have 8 phases as shown in Figure 9.

If we want to identify and control the spillover on $L_{1,2}$, we should take Phase 2 as the phase whose green light should be truncated when spillover is detected and Phase 1 as the phase which can obtain more green time to explain our method. According to the original timing plan provided by the modelbased control method in the current cycle, we can infer the time when the green time for Phase 2 is started. When Phase 2 (terminating phase) is green, the potential queue spillover on $L_{1,2}$ will be identified. The spillover control controller will work if a possible spillover is detected. The flowchart of the spillover control for Intersection 1 is shown in Figure 7. The spillover controller will first execute ECG to adjust the signal light state of Phase 2 to red to prevent possible spillover. The remaining green time can be reassigned to Phase 1 if the remaining green time is longer than the sum of $g_{\min }$ and amber time. When the queue dissipation is detected, RUG will be executed to adjust the signal light state of Phase 1 and Phase 2 to green and to red, respectively, if the remaining passing time is enough. Similarly, in order to prevent possible spillover on link $L_{1,4}$ the control scheme of Phase 6 and Phase 5 can be concluded. There is no need to take control of Phases $3,4,7$, and 8 due to the traffic flow volume is low on these phases.

\section{Case Study}

6.1. Simulation Settings. We evaluate the results of the proposed method in the above virtual network as shown in Figure 8 . The total time to simulate the road network is set as 


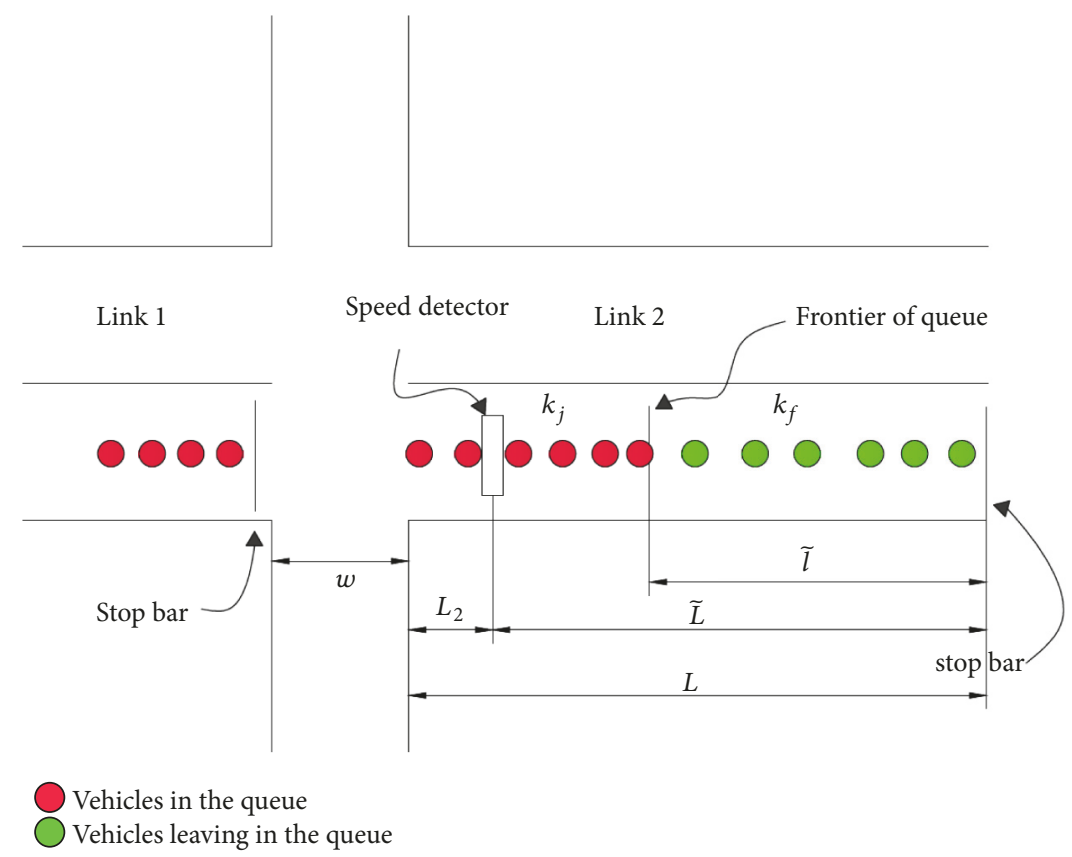

FiguRE 6: Queue dissipation identification based on speed detector. Speed detector is deployed to detect the speed when vehicles pass over it.

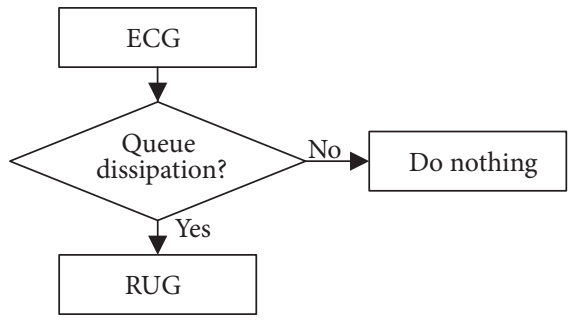

FIGURE 7: The flowchart of queue spillover control on link $L_{1,2}$. ECG is activated to Early Cut-Off Green time of the phase encountering spillover. RUG is activated to return the unused green time the phase whose green time is Early Cut-Off.

one hour. We set the time that the simulation will run before starting to collect results as three signal cycles. Relevant parameters and network configurations will be introduced in the following.

Intersection 1 is the studied intersection in this paper. The intersection adjacent to Intersection 1 is Intersection 2 . All the links of the simulation network have three lanes where lane 1 , lane 2 , and lane 3 which are numbered from left to right on each road represent left-turn lane, through lane, and through right-turn lane, respectively. The VISSIM4.3 is selected as the simulation tool to simulate traffic movements and provide evaluation results to determine the performance of different traffic signal methods. Python provides the output of control parameter of green time splits based on the traffic flow data collected by the corresponding detectors. The saturation flow rate $\mu_{u, d}$ for all links is 2000 vehicle/h. $g_{\min }$ and $g_{\max }$ for all intersections are set as $10 \mathrm{~s}$ and $50 \mathrm{~s}$, respectively. $c_{c y c l e}$ is set as $140 \mathrm{~s}$ which is equal to the sampling time. The simulation results are based on $N_{p}=5 . L_{1,2}$ is set as 200 meters.

In order to obtain the results with different traffic signal control methods, we should provide traffic volume from different entries. Table 1 shows the Traffic volume for Intersection 1 during the simulation duration. NB, SB, EB, and $\mathrm{WB}$ represent the entrance of north, south, east, and west, respectively, for Intersection 1 .

Table 2 lists the parameters used in IGAABC. The trials limit represents the times for which the food source is not updated is set as 100; both the numbers of employed bees and onlooker bees are equal to 15 ; the crossover probability is set as 0.6 and mutation probability is 0.01 ; the number of iterations is 49 .

We select the average vehicle delay for each phase at the studied intersection and the maximum queue length on link $L_{1,2}$ obtained by the simulation evaluation of VISSIM4.3 as the evaluation criterion. The result comparison will be described in detail in the following section.

6.2. Simulation Results. In this section, the simulation evaluation data of delay and queue length collected from VISSIM4.3 is provided to evaluate the performance of traffic signal control. The proposed control method will be compared with the methods of model-based predictive control and adaptive signal control. In addition, the proposed control method with our improved ABC algorithm will be compared with the method with $\mathrm{ABC}$ algorithm to verify the performance of our algorithm. The detailed descriptions of different traffic signal control methods are introduced as below:

(1) Model-based predictive control, whose green time splits for all intersections are optimized according to the measured traffic flow state is showed. 


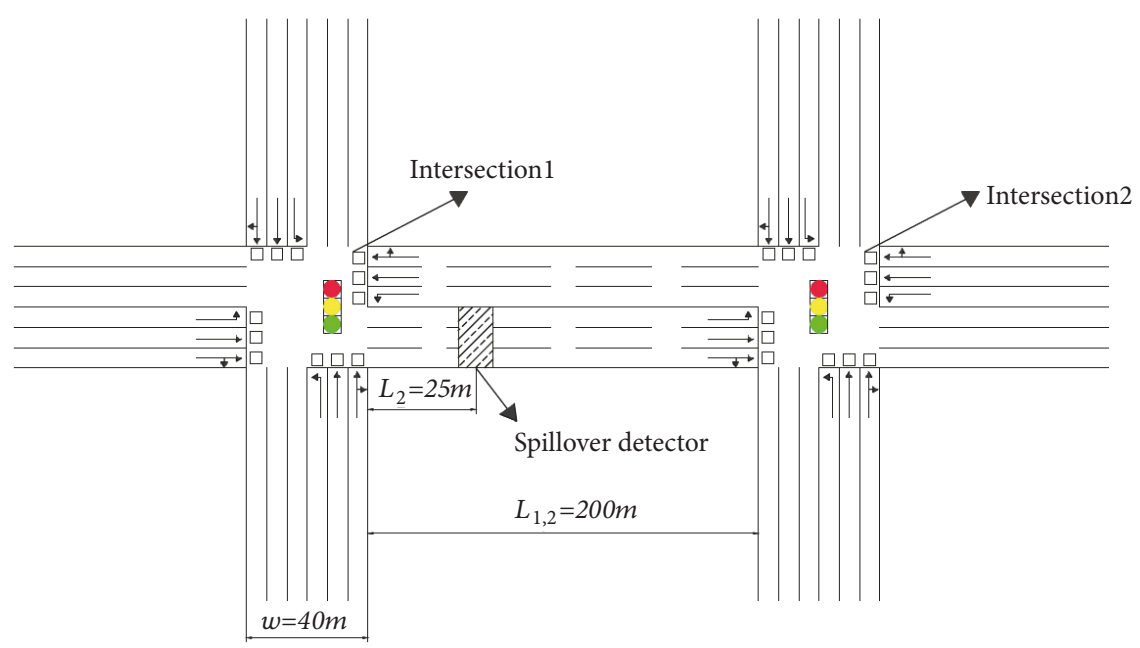

FIGURE 8: Road network Layout for the simulation. The adjacent intersection is taken into account to simulate the practical road traffic condition.

TABLE 1: Traffic volume for Intersection 1 during the simulation duration.

\begin{tabular}{lccc}
\hline ENTRY & Left Turn[vehicle/h] & Through[vehicle/h] & Right Turn[vehicle/h] \\
\hline NB & 147 & 256 & 166 \\
SB & 181 & 221 & 168 \\
EB & 166 & 1297 & 215 \\
WB & 175 & 963 & 183 \\
\hline
\end{tabular}

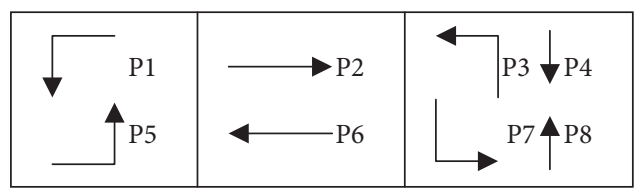

Figure 9: The design of phase sequence. P1 and P5 are grouped into signal group 1. P2 and P6 are grouped into signal group 2. The residual phases are grouped into signal group 3.

TABLE 2: IGAABC parameters settings.

\begin{tabular}{lcc}
\hline Parameter & Description & value \\
\hline limit & trials_limit & 100 \\
SN & The number of employed bees & 15 \\
& The number of onlooker bee & \\
$p_{c}$ & Crossover probability & 0.6 \\
$p_{m}$ & Mutation probability & 0.01 \\
nruns & Number of iterations & 49 \\
\hline
\end{tabular}

(2) Adaptive signal control, which can adaptively adjust the green time according to the real-time traffic condition to prevent the possible queue spillover [23]. The basic green time splits scheme of each intersection consists of three signal groups. As shown in Figure 10, the green time splits of the three signal groups at Intersection 1 are $35 \mathrm{~s}, 65 \mathrm{~s}$, and $40 \mathrm{~s}$, respectively, while the green time splits of the three signal groups for Intersection 2 are 45 s, 55 s, and 40 s, respectively.
TABLE 3: Total average vehicle delay at intersection 1 with different control schemes.

\begin{tabular}{lc}
\hline Signal Control Method & Average Vehicle Delay [s] \\
\hline Model-based predictive control & 535.1 \\
Adaptive signal control & 525.3 \\
Proposed method (ABC) & 479.3 \\
Proposed method (IGAABC) & 464.6 \\
\hline
\end{tabular}

(3) Proposed method (ABC), whose control method is based on the proposed improved model-based predictive control method in this paper and optimization algorithm is based on the basic ABC algorithm.

(4) Proposed method (IGAABC), whose control method is based on the proposed improved model-based predictive control method, while the optimization algorithm of this method is based on the improved hybrid artificial bee colony algorithm IGAABC proposed in the paper.

Table 3 shows the total average vehicle delay at Intersection 1 with different methods. We can conclude that the delay in proposed method (ABC) method can be decreased by 8.8 percentage compared with adaptive signal control [23] method and 10.4 percentage compared with the model-based predictive control. What is more, the delay in proposed method (IGAABC) method can be decreased by 11.6 percentage compared with adaptive signal control method and 13.2 percentage compared with the model-based 


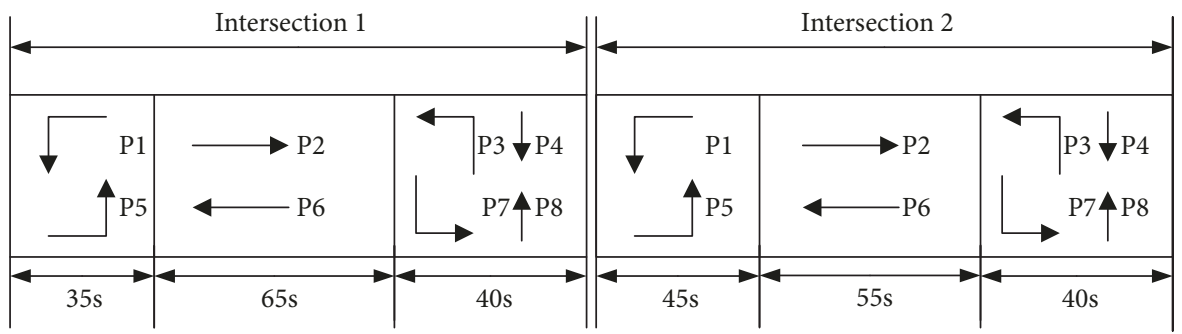

FIGURE 10: The basic green time splits scheme at different intersections. The green time splits of the three signal groups at Intersection 1 are $35 \mathrm{~s}, 65 \mathrm{~s}$, and $40 \mathrm{~s}$, respectively, while the green time splits of the three signal groups for Intersection 2 are $45 \mathrm{~s}, 55 \mathrm{~s}$, and $40 \mathrm{~s}$, respectively.

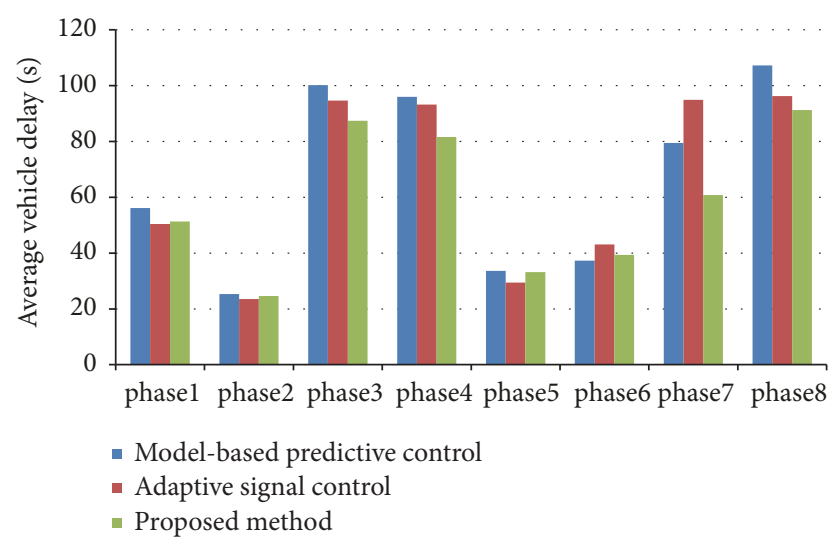

FIGURE 11: Average vehicle delay for each phase with different control schemes. The simulation time is one hour with the first three cycles as warm-up time.

predictive control. Therefore, the improved hybrid artificial bee colony algorithm IGAABC improves the performance of basic artificial bee colony algorithm by 3.1 percentage in terms of average vehicle delay. The simulation results verify that the proposed method (IGAABC) method can incredibly promote efficiency in traffic signal control and the solutions obtained by the optimization algorithm of IGAABC are better than the basic $\mathrm{ABC}$ algorithm. The average vehicle delay for different control scheme at intersection 1 is shown in Table 3 in detail.

The detailed comparisons of average vehicle delay for each phase at intersection 1 are shown in Figure 11. Due to different signal timing schemes that can be derived by the proposed method and model-based predictive control method according to different traffic flow conditions, it is more flexible to control the changing traffic flow to balance the flow in a road network. Although the differences for Phase 2 and Phase 6 between the proposed method and the modelbased predictive control are not apparent, the delay of other phases can be decreased significantly because of the strategy of queue spillover control. By comparing the proposed method in this paper with adaptive signal control method, we can conclude that the delay for most phases at Intersection 1 will be decreased, which would be of great benefit to the drivers who are waiting at the corresponding intersection.

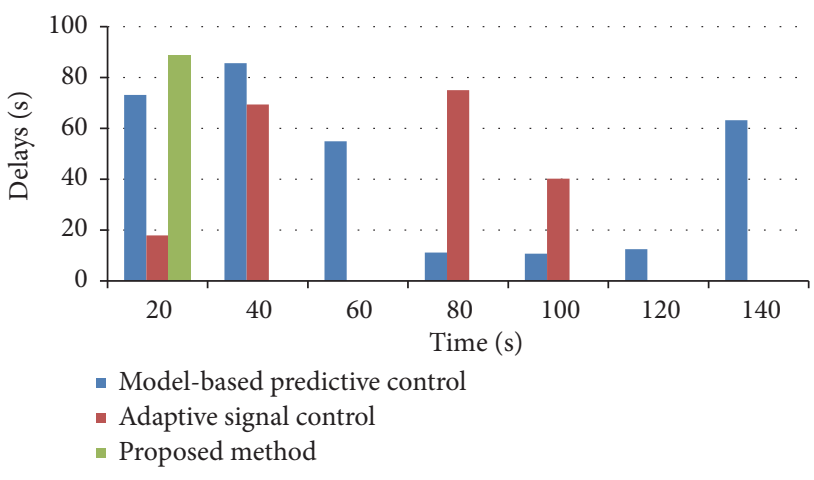

Figure 12: Delay of Phase 2 in a cycle. The delay is evaluated in a specific cycle whose simulation time ranging from $2300 \mathrm{~s}$ to $2440 \mathrm{~s}$.

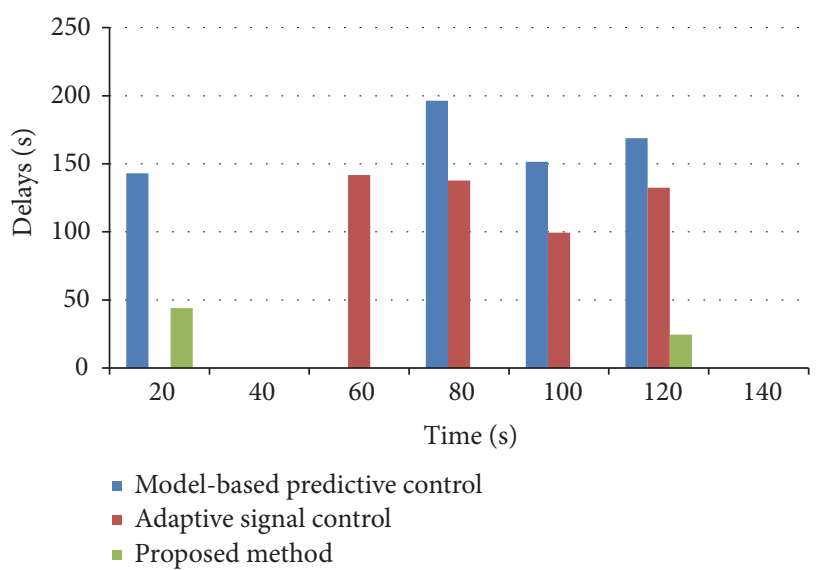

Figure 13: Delay of Phase 7 in a cycle. The delay is evaluated in a specific cycle whose simulation time ranging from $2300 \mathrm{~s}$ to $2440 \mathrm{~s}$.

The results further demonstrate that our proposed method is superior to adaptive signal control method in terms of delay.

We further study the delay in a specific cycle whose simulation time ranging from $2300 \mathrm{~s}$ to $2440 \mathrm{~s}$, where queue spillover will happen frequently. As shown in Figures 12 and 13, we take the delay of Phase 2 and Phase 7, for example. We can conclude that both the adaptive signal control method and the proposed method can efficiently deal with the traffic signal control problem to decrease the delay when the traffic demand of the studied intersection is significantly huge during peak hours, while the model-based predictive control 


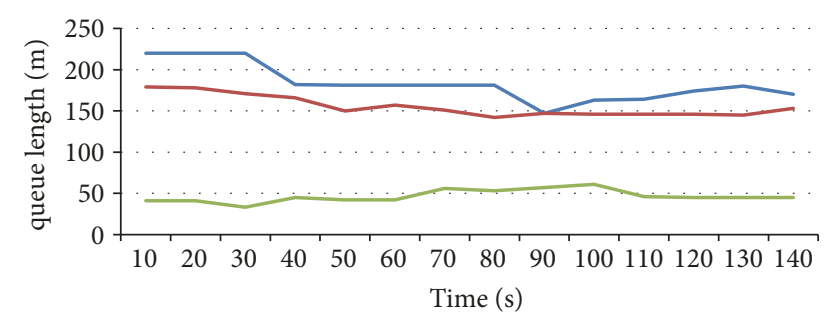

- Model-based predictive control
- Adaptive signal control
Proposed method

FIGURE 14: Maximum queue length on link $L_{1,2}$. Queue length can demonstrate the control performance to decrease traffic volume on a specific road.

TABLE 4: The CPU time of the integrated method with different prediction horizons.

\begin{tabular}{lccc}
\hline Prediction Horizon & Average[s] & Maximum[s] & Minimum[s] \\
\hline $\mathrm{NP}=3$ & 39.09 & 40.99 & 37.38 \\
$\mathrm{NP}=5$ & 51.45 & 56.59 & 48.56 \\
$\mathrm{NP}=7$ & 81.28 & 86.44 & 77.48 \\
\hline
\end{tabular}

cannot control the studied intersection well which leads to a large delay over time. However, both the proposed method and the model-based predictive control method can adjust the green timing scheme according to the real-time traffic condition, which would facilitate balancing the traffic flow on each road. Therefore, the delay will be controlled according to the change of traffic flow state based on these two control method.

According to the simulation results, the proposed method and the adaptive signal control method can both maintain the queue length less than the length of $L_{1,2}(200 \mathrm{~m})$ to avoid spillover under oversaturated traffic condition, while the model-based predictive control cannot avoid spillover on link $L_{1,2}$. As shown in Figure 14, we choose the same cycle which suffers a frequent occurrence of the spillover. The adaptive signal control method and the proposed signal control method will take actions to prevent the spillover when they detect the possible queue spillover. The proposed signal control method can adjust the green time splits according to the changing traffic flow; thus it can maintain the queue length at a relatively low level. Although the model-based predictive control method will optimize the green time splits according to the measured traffic flow, it cannot prevent traffic congestion when the queue spillover occurs. Therefore, the queue under oversaturated conditions will be at a high level. The simulation results verify that the proposed method outperforms other methods in delay and maintains the queue length in a specific range.

The proposed method will spend some time on solving the optimal solutions of green time splits. Table 4 shows the CPU time of the proposed method with different prediction horizons which are equal to 3,5 , and 7 respectively. The time units in Table 4 are second. Average, Maximum, and
Minimum denotes the average time, maximum time, and minimum time for a run, respectively, to obtain the optimal time splits in a control step. The time of a control step is set to one signal control cycle which equals $140 \mathrm{~s}$. The CPU time shown in Table 4 for different prediction horizon is less than $140 \mathrm{~s}$. Therefore, the green time splits can be obtained in time in the proposed method of this paper.

In a word, the proposed method can significantly improve the overall performance of the studied intersection. The proposed signal control method can not only provide appropriate timing plan according to the changing traffic flow, but also it can avoid the occurrence of queue spillover under oversaturated traffic condition. Therefore this method can benefit these two ideas to reduce the delay and improve the efficiency of traffic control.

6.3. Sensitivity Analysis. There are many factors which may affect the performance of signal optimization. Given different traffic volumes, turning movement changes, and speed limits of the road links, the simulation results which can reflect the performance of signal optimization may be different. Li et al. [40] modeled left-turn driving behavior to reveal the impact of different degrees of conflict on the performance of signal optimization under mixed traffic conditions. Considering the study in this paper is based on the assumption that traffic flow merely consists of single-motorized vehicle, the impact of degrees of conflict on signal optimization is neglected. Therefore, traffic volumes, turning movement changes, and speed limits of the road links are selected to evaluate the sensitivity of proposed method. The total delay which can reflect the signal control effect at the studied intersection is regarded as the performance index.

Sensitivity analysis under different traffic volume conditions includes 6 traffic scenarios. We select different factors $\mathrm{m}$ which are $0.2,0.4,0.6,0.8,1.0$, and 1.2 of the traffic volume shown in Table 1, respectively, to simulate different traffic scenarios. As shown in Table 5, we can conclude that the total delay of proposed method and model-based predictive control method can be maintained at a low level when the value of $\mathrm{m}$ is no larger than 0.4 which simulates an unsaturated traffic condition. However, with an increase of the value of $m$ which reflects an increasing traffic demand, the performance of the basic model-based predictive control method is apparently worse than the proposed method and adaptive signal control method. The percentage in parentheses represents the percentage of performance improvement by the proposed method compared with a specified method. The proposed methods can always perform well no matter what traffic condition it is. The improvement rate of performance is shown in Table 5.

Sensitivity analysis under different turning movement changes includes 3 scenarios. We assume that $\beta=[L, T, R]$, where L,T,R represents the percentages of left-turn, through, and right-turn movements, respectively. The simulation results are shown in Table 6 . Although total delay of proposed method with the value of $\beta$ which is equal to $[1,1,1]$ or $[2,1,1]$ is no better than adaptive signal control method, it performs well when the value of $\beta$ is set as $[1,2,1]$ which 
TABLE 5: Total delay at Intersection 1 with different traffic scenarios.

\begin{tabular}{lccc}
\hline Traffic volume factor & Proposed method & Model-based predictive control & Adaptive signal control \\
\hline 0.2 & 380 & $396.1(+4.1 \%)$ & $424.7(+10.5 \%)$ \\
0.4 & 436.2 & $442.3(+1.38 \%)$ & $452.5(+3.6 \%)$ \\
0.6 & 442.5 & $506.4(+12.62 \%)$ & $482.1(+8.2 \%)$ \\
0.8 & 452.8 & $525.1(+13.77 \%)$ & $517.5(+12.5 \%)$ \\
1 & 469.4 & $535.1(+12.28 \%)$ & $525.3(+10.64 \%)$ \\
1.2 & 492.4 & $656.8(+25.03 \%)$ & $642.4(+23.35 \%)$ \\
\hline
\end{tabular}

TABLE 6: Total delay at Intersection 1 with different turning movement changes.

\begin{tabular}{lccc}
\hline$\beta$ & {$[1,1,1]$} & {$[1,2,1]$} & {$[2,1,1]$} \\
\hline $\begin{array}{l}\text { Proposed method } \\
\begin{array}{l}\text { Model-based } \\
\text { predictive control }\end{array}\end{array}$ & 514 & 441.8 & 533.7 \\
$\begin{array}{l}\text { Adaptive signal } \\
\text { control }\end{array}$ & 1009 & 792.5 & 996.3 \\
\hline
\end{tabular}

TABLE 7: Total delay at Intersection 1 with different speed limits of the road links.

\begin{tabular}{lccc}
\hline speed limits & 30 & 50 & 70 \\
\hline $\begin{array}{l}\text { Proposed method } \\
\begin{array}{l}\text { Model-based } \\
\text { predictive control }\end{array}\end{array}$ & 465.3 & 469.4 & 742.1 \\
$\begin{array}{l}\text { Adaptive signal } \\
\text { control }\end{array}$ & 499.9 & 535.1 & 1302.9 \\
\hline
\end{tabular}

can reflect the turning movement in real urban road where the percentage of through movement is larger than another movement. Therefore, the proposed method is available in practice.

Sensitivity analysis under different speed limits of the road links is shown in Table 7 . The units for the speed limits in Table 7 are $\mathrm{km} / \mathrm{h}$. The units for the total delay in Table 7 are second. The performance of proposed method performs well when the speed limits of the road links are less than 70 $\mathrm{km} / \mathrm{h}$. However, the total delay of proposed method increases slightly when the speed limit is set as $70 \mathrm{~km} / \mathrm{h}$. Considering that the speed under oversaturated traffic conditions will not be too high, the proposed method is effective to deal with the problem of traffic signal control when traffic demand is high.

\section{Conclusion and Future Outlook}

To summarize, our proposed method can deal with the problem of traffic signal control at intersections when capacity of the system cannot satisfy the demand under oversaturated traffic conditions. We propose an improved model-based predictive control approach which can predict traffic demand over a finite horizon according to the measured traffic flow information to calculate the optimal green time splits plan with the objective of total travel time minimum and avoid queue spillover under oversaturated traffic conditions. Moreover, to solve complicated optimization problems in a short time which contributes to online applications, we propose an improved hybrid artificial bee algorithm to address the optimal control problem of the proposed improved modelbased predictive control approach as soon as possible. The simulation results verified that our proposed method can manage queue effectively and balance the traffic flow on different roads simultaneously which can improve the performance of the intersection significantly.

Other subjects will be explored in the future. We can first consider the following aspects including how to optimize traffic control along trunk roads or a huge network under oversaturated condition. Second, we can study the algorithm to solve the optimization model to obtain the solutions as soon as possible. Finally, the proposed method is based on a fixed cycle and phase sequence which limits the application in practical urban traffic condition.

\section{Data Availability}

The traffic volume data used to support the findings of this study are included within the article. Table 1 provides the traffic volume information, which we regard as the traffic flow input of each entrance at our studied intersection. These data can be applied to simulation tool VISSIM4.3 to obtain the comparison results with different traffic signal control methods.

\section{Conflicts of Interest}

The authors declare that they have no conflicts of interest.

\section{Acknowledgments}

This work was financially supported by Chinese National Science Foundation (61572165) and Projects of Zhejiang Province (LGF18F030006).

\section{References}

[1] L. Qi, M. C. Zhou, and W. J. Luan, "Modeling and control of urban road intersections with incidents via timed Petri nets," in Proceedings of the 12th IEEE International Conference on Networking, Sensing and Control (ICNSC), pp. 185-190, IEEE, 2015.

[2] V. Valls, J. Monteil, and M. Bouroche, "A convex optimisation approach to traffic signal control," in Proceedings of the 19th IEEE International Conference on Intelligent Transportation Systems (ITSC), pp. 1508-1515, IEEE, 2016. 
[3] S. Li, G. Wang, T. Wang et al., "Study on the reduced traffic congestion method based on dynamic guidance information," Communications in Theoretical Physics, vol. 69, no. 5, article 577, 2018.

[4] J. Wu, B. Chen, K. Zhang et al., "Ant pheromone route guidance strategy in intelligent transportation systems," Physica A: Statistical Mechanics and its Applications, vol. 503, pp. 591-603, 2018.

[5] Z. Sun, "Continuous transportation network design problem based on bi-level programming model," Procedia Engineering, vol. 137, pp. 277-282, 2016.

[6] L. Han, H. Sun, D. Z. W. Wang et al., "The combination of continuous network design and route guidance," Computers \& Operations Research, vol. 73, pp. 92-103, 2016.

[7] H. Ding, F. Guo, X. Zheng et al., "Traffic guidance-perimeter control coupled method for the congestion in a macro network," Transportation Research Part C: Emerging Technologies, vol. 81, pp. 300-316, 2017.

[8] Q. He, R. Kamineni, and Z. Zhang, "Traffic signal control with partial grade separation for oversaturated conditions," Transportation Research Part C: Emerging Technologies, vol. 71, pp. 267-283, 2016.

[9] C. C. Liu, "Bandwidth-constrained delay optimization for signal systems," ITE Journal, vol. 58, no. 12, pp. 21-26, 1988.

[10] S. Samra, A. El-Mahdy, and Y. Wada, "A linear time and space algorithm for optimal traffic-signal duration at an intersection," IEEE Transactions on Intelligent Transportation Systems, vol. 16, no. 1, pp. 387-395, 2015.

[11] G. B. Castro, A. R. Hirakawa, and J. S. C. Martini, "Adaptive traffic signal control based on bio-neural network," Procedia Computer Science, vol. 109, pp. 1182-1187, 2017.

[12] L. Zhihui, C. Qian, Z. Yonghua et al., "Signal cooperative control with traffic supply and demand on a single intersection," IEEE Access, vol. 6, pp. 54407-54416, 2018.

[13] C. F. Daganzo, "Queue spillovers in transportation networks with a route choice," Transportation Science, vol. 32, no. 1, pp. 3-11, 1998.

[14] D. C. Gazis, "Optimum control of a system of oversaturated intersections," Operations Research, vol. 12, no. 6, pp. 815-831, 1964.

[15] P. G. Michalopoulos and G. Stephanopoulos, "Oversaturated signal systems with queue length constraints-I: single intersection," Transportation Research, vol. 11, no. 6, pp. 413-421, 1977.

[16] W. Xiang, J. Xiao, and Y. Jiang, "Real-time signalization for an oversaturated intersection via static state feedback control: a switched system approach," Journal of The Franklin Institute, vol. 352, no. 8, pp. 3304-3324, 2015.

[17] W. Sun, Y. Wang, G. Yu et al., "Quasi-optimal feedback control for an isolated intersection under oversaturation," Transportation Research Part C: Emerging Technologies, vol. 67, pp. 109-130, 2016.

[18] W. Lin, H. K. Lo, and L. Xiao, "A quasi-dynamic robust control scheme for signalized intersections," Journal of Intelligent Transportation Systems: Technology, Planning, and Operations, vol. 15, no. 4, pp. 223-233, 2011.

[19] D. Ma, D. Wang, Y. Bie et al., "A method of signal timing optimization for spillover dissipation in urban street networks," Mathematical Problems in Engineering, vol. 2013, Article ID 580546, 9 pages, 2013.

[20] H. Qi, Y. Ye, J. Xu et al., "Intersection control considering channelized section spillback using a flexible phase plan," Journal of Transportation Engineering, vol. 142, no. 1, article 04015032, 2015.
[21] C. Osorio, X. Chen, M. Marsico et al., "Reducing gridlock probabilities via simulation-based signal control," Transportation Research Procedia, vol. 6, pp. 101-110, 2015.

[22] W. Sun, Y. Wang, G. Yu et al., "Quasi-optimal feedback control for a system of oversaturated intersections," Transportation Research Part C: Emerging Technologies, vol. 57, pp. 224-240, 2015.

[23] Y. Ren, Y. Wang, G. Yu et al., "An adaptive signal control scheme to prevent intersection traffic blockage," IEEE Transactions on Intelligent Transportation Systems, vol. 18, no. 6, pp. 1519-1528, 2017.

[24] C. Ren, W. Zhang, L. Qin et al., "Queue spillover management in a connected vehicle environment," Future Internet, vol. 10, no. 8, article 79, 2018.

[25] C. Wu, X. Zeng, J. Zhan et al., "Study on optimal model of traffic signal control at oversaturated intersection," in Proceedings of the International Symposium for Intelligent Transportation and Smart City, pp. 28-38, Springer, Singapore, 2019.

[26] K. Aboudolas, M. Papageorgiou, A. Kouvelas, and E. Kosmatopoulos, "A rolling-horizon quadratic-programming approach to the signal control problem in large-scale congested urban road networks," Transportation Research Part C: Emerging Technologies, vol. 18, no. 5, pp. 680-694, 2010.

[27] S. Lin, B. de Schutter, Y. Xi, and H. Hellendoorn, "Efficient network-wide model-based predictive control for urban traffic networks," Transportation Research Part C: Emerging Technologies, vol. 24, pp. 122-140, 2012.

[28] Z. Zhou, B. De Schutter, S. Lin, and Y. Xi, "Two-level hierarchical model-based predictive control for large-scale urban traffic networks," IEEE Transactions on Control Systems Technology, vol. 25, no. 2, pp. 496-508, 2017.

[29] S. Lin, B. De Schutter, Y. Xi et al., "A simplified macroscopic urban traffic network model for model-based predictive control," IFAC Proceedings, vol. 42, no. 15, pp. 286-291, 2009.

[30] S. U. Mane, R. Omane, and A. Pawar, "GPGPU based teaching learning based optimization and artificial bee colony algorithm for unconstrained optimization problems," in Proceedings of the 5th IEEE International Advance Computing Conference (IACC), pp. 1056-1061, IEEE, 2015.

[31] D. Karaboga and B. Basturk, "Artificial bee colony (ABC) optimization algorithm for solving constrained optimization problems," in Proceedings of the International fuzzy systems association world congress, pp. 789-798, Springer, Berlin, Heidelberg, Germany, 2007.

[32] C. Zhang, D. Ouyang, and J. Ning, "An artificial bee colony approach for clustering," Expert Systems with Applications, vol. 37, no. 7, pp. 4761-4767, 2010.

[33] A. Jovanović, M. Nikolić, and D. Teodorović, "Area-wide urban traffic control: a bee colony optimization approach," Transportation Research Part C: Emerging Technologies, vol. 77, pp. 329350, 2017.

[34] G. Zhu and S. Kwong, "Gbest-guided artificial bee colony algorithm for numerical function optimization," Applied Mathematics and Computation, vol. 217, no. 7, pp. 3166-3173, 2010.

[35] W. F. Gao and S. Y. Liu, "Improved artificial bee colony algorithm for global optimization," Information Processing Letters, vol. 111, no. 17, pp. 871-882, 2011.

[36] W. Ma, Z. Sun, J. Li, M. Song, and X. Lang, "An improved artificial bee colony algorithm based on the strategy of global reconnaissance," Soft Computing, vol. 20, no. 12, pp. 4825-4857, 2016. 
[37] M. Zhang and K. Wu, "An improved genetic algorithm for the re-entrant and flexible job-shop scheduling problem," in Proceedings of the Chinese Control and Decision Conference (CCDC), pp. 3399-3404, IEEE, 2016.

[38] Y. Li, R. Wang, Y. Liu et al., "Satellite range scheduling with the priority constraint: An improved genetic algorithm using a station ID encoding method," Chinese Journal of Aeronautics, vol. 28, no. 3, pp. 789-803, 2015.

[39] H. Li, N. Chen, L. Qin et al., "Queue length estimation at signalized intersections based on magnetic sensors by different layout strategies," Transportation Research Procedia, vol. 25, pp. 1629-1647, 2017.

[40] H. Li, S. Li, H. Li et al., "Modeling left-turn driving behavior at signalized intersections with mixed traffic conditions," Mathematical Problems in Engineering, vol. 2016, Article ID 4015271, 11 pages, 2016. 


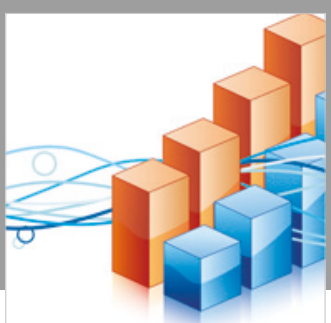

Advances in

Operations Research

\section{-n-m}
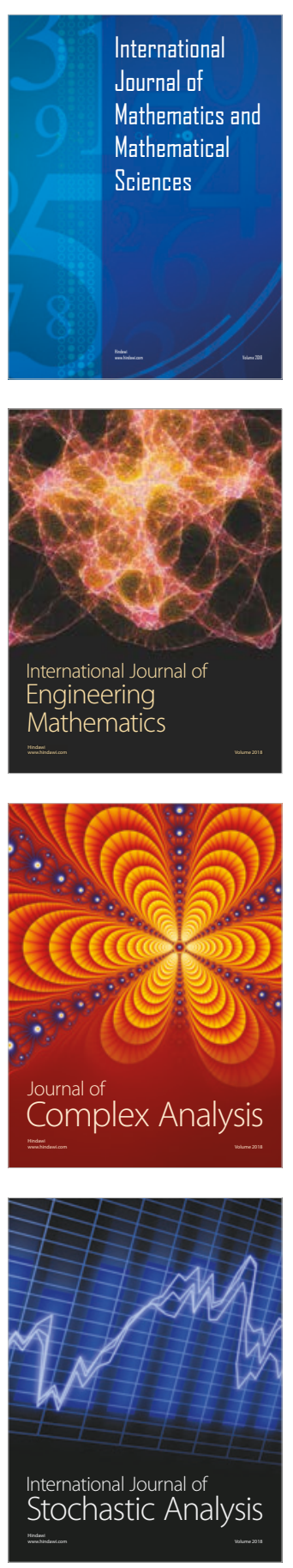
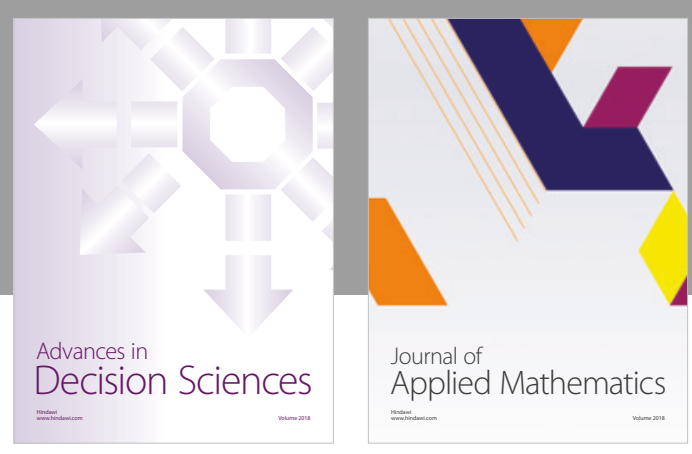

Journal of

Applied Mathematics
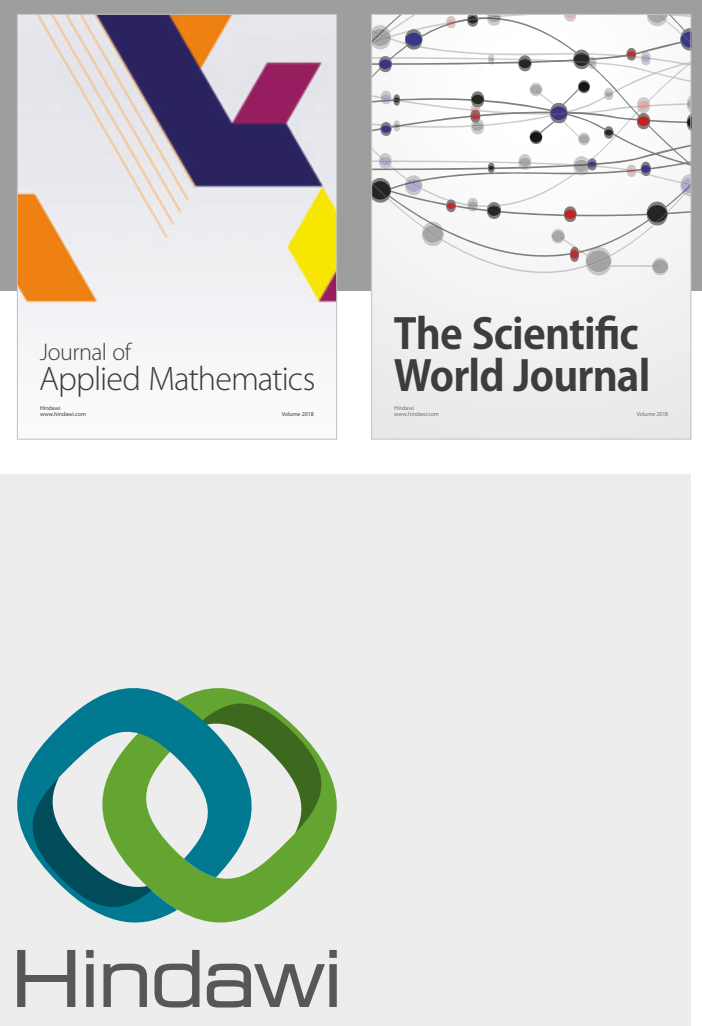

Submit your manuscripts at

www.hindawi.com

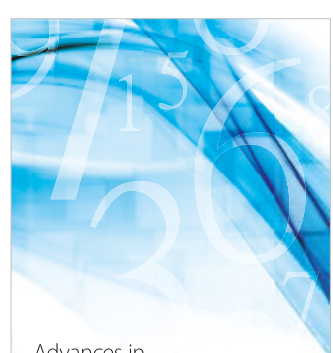

Advances in
Numerical Analysis
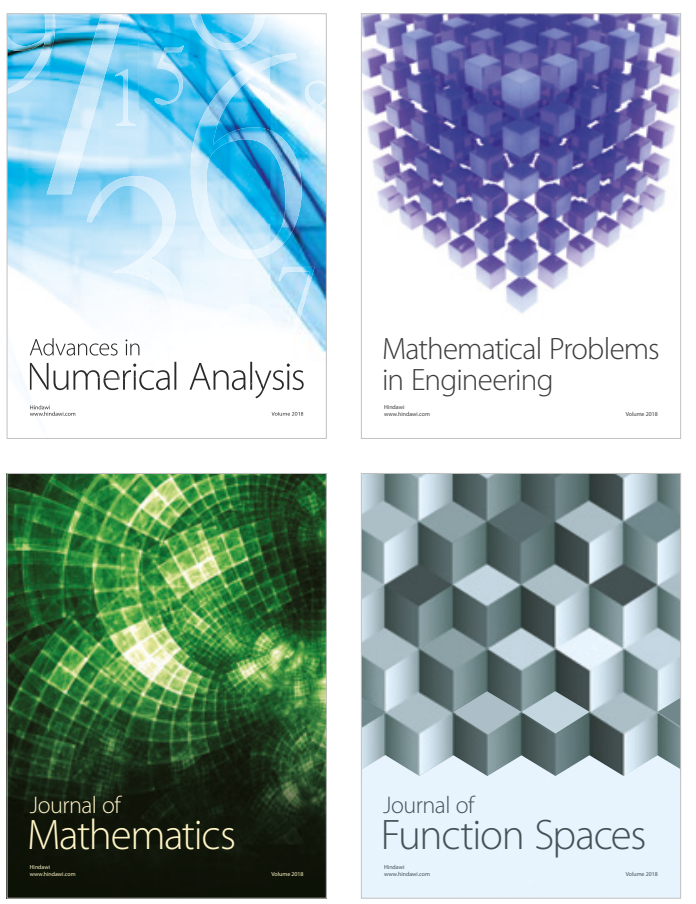

Mathematical Problems in Engineering

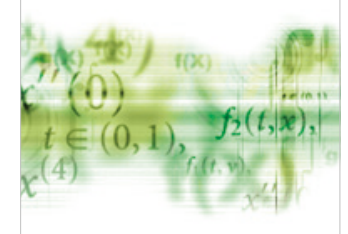

International Journal of

Differential Equations

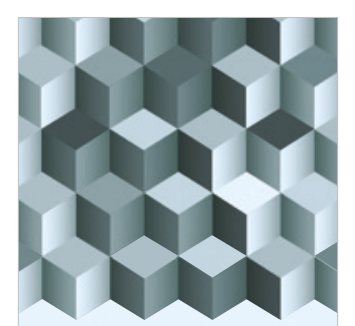

Journal of

Function Spaces
The Scientific

World Journal

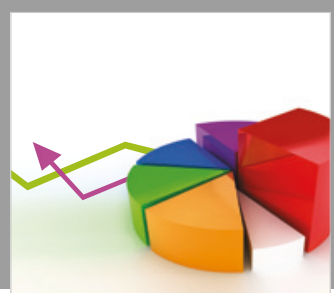

Journal of

Probability and Statistics
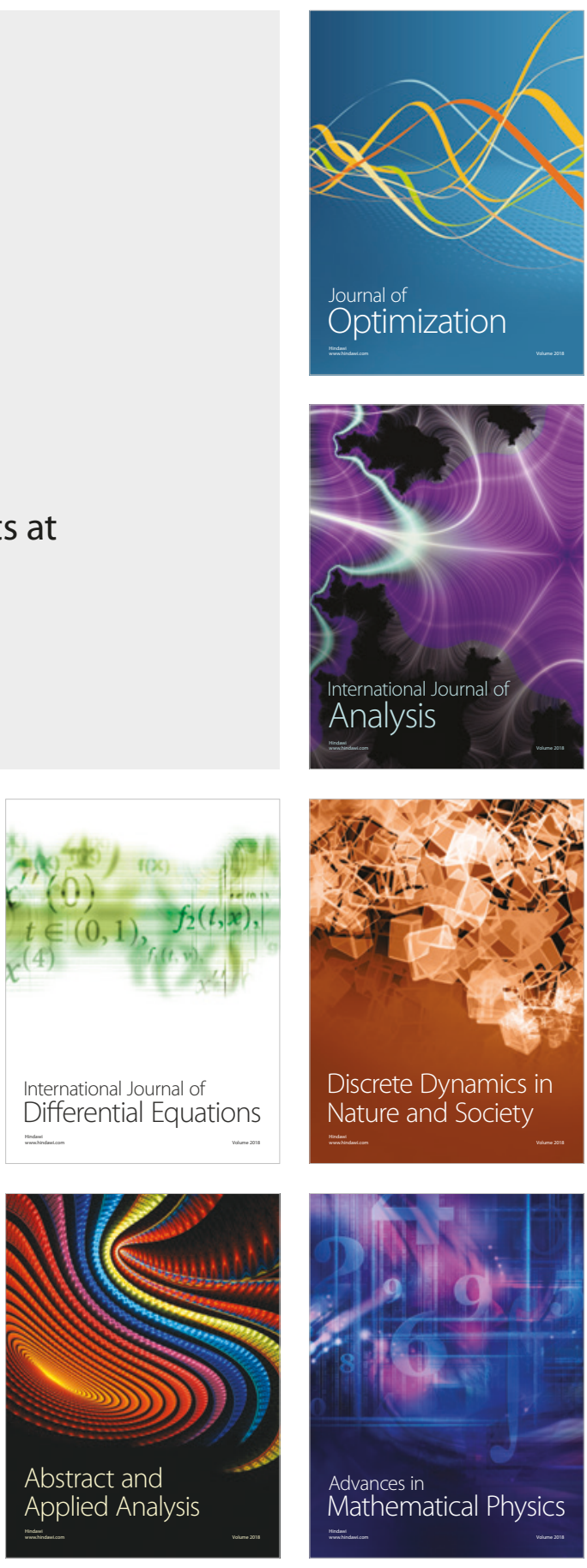